\title{
COMBINATORIAL LEMMAS FOR NONORIENTED PSEUDOMANIFOLDS
}

\author{
AdAm IDZIK - Konstanty JunOSZA-SzaniaWski
}

\begin{abstract}
Sperner lemma type theorems are proved for nonoriented primoids and pseudomanifolds. A rank function of a primoid is defined. Applications of these theorems to the geometric simplex are given. Also KnasterKuratowski-Mazurkiewicz type theorems on covering of the geometric simplex are presented.
\end{abstract}

\section{Introduction}

One of the fundamental theorems in nonlinear analysis is the Sperner lemma ([19], [5]). It has been applied to prove the Knaster-Kuratowski-Mazurkiewicz theorem on covering of the geometric simplex ([13], [20]) and the Brouwer fixed point theorem ([4]). Extensions of the Sperner lemma were presented by Fan ([6]-[8]) for $n$-pseudomanifolds, by Shapley ([18]) for multivalued labellings and by Bapat ([2], [3]) for oriented primoids and $n$ coverings. Dual theorems to the Sperner lemma are the Alexandroff and Pasynkoff theorem ([1], see also [20]) and the Ichiishi theorem ([10]). In this paper we generalize many of these theorems and derive new results on coverings of the geometric simplex.

2000 Mathematics Subject Classification. Primary 05B30,47H10; Secondary 52A20,54H25.

Key words and phrases. KKM covering, labelling, primoid, pseudomanifold, simplicial complex. 


\section{Preliminaries}

By $\mathbb{N}, \mathbb{R}$ we denote the set of natural numbers and reals, respectively. Let $n \in \mathbb{N}$ and $V$ be a finite set of the cardinality at least $n+1 . \quad \mathbf{P}(V)$ is the family of all subsets of $V$ and $\mathbf{P}_{n}(V)$ is the family of all subsets of $V$ of the cardinality $n+1$. An element of $\mathbf{P}_{n}(V)$ is called $n$-simplex over the set $V$. Let $\mathbf{C}$ be a nonempty family of $n$-simplexes over $V$. We call $\mathbf{C}$ an $n$-complex over the set $V$.

Let $A=\left\{a_{0}, \ldots, a_{m}\right\} \subset \mathbb{R}^{n}$ be a set of points $(m \in \mathbb{N})$. Then co $A=$ $\left\{\alpha_{0} a_{0}+\ldots+\alpha_{m} a_{m}: \sum_{i=0}^{m} \alpha_{i}=1, \alpha_{i} \geq 0\right.$ for $\left.i \in\{0, \ldots, m\}\right\}$ is a convex hull of $A$, af $A=\left\{\alpha_{0} a_{0}+\ldots+\alpha_{m} a_{m}: \sum_{i=0}^{m} \alpha_{i}=1, \alpha_{i} \in \mathbb{R}\right.$ for $\left.i \in\{0, \ldots, m\}\right\}$ is an affine hull of $A$, ri $Z$ is the relative interior of a set $Z \subset \mathbb{R}^{n}$ and bd $Z$ is the boundary of a set $Z \subset \mathbb{R}^{n}$. A finite set $A=\left\{a_{0}, \ldots, a_{m}\right\} \subset \mathbb{R}^{n}$ is affinely independent if the dimension of af $A$ is $m(m \leq n)$. We denote $N=\{0, \ldots, n\}$.

\section{Primoids}

Let $U$ be a finite set of the cardinality at least $n+1$. An $n$-primoid $\mathbf{L}_{n}^{U}$ over $U$ is a nonempty family of $n$-simplexes over $U$ fulfilling the following condition: for every $n$-simplex $T \in \mathbf{L}_{n}^{U}$ and for any $u \in U$ there exists exactly one $u^{\prime} \in T$ such that an $n$-simplex $T \backslash\left\{u^{\prime}\right\} \cup\{u\} \in \mathbf{L}_{n}^{U}$. n-simplexes belonging to $\mathbf{L}_{n}^{U}$ are called complete. For brevity we write $\mathbf{L}_{n}$ instead of $\mathbf{L}_{n}^{U}$.

The $n$-primoid $\mathbf{L}_{n}$ can be also defined by the family of $n$-simplexes over $U$ such that every $(n+1)$-simplex over $U$ contains either none or two $n$-simplexes belonging to the $n$-primoid $\mathbf{L}_{n}$.

EXAmple 3.1. Let $U=N$. $\mathbf{L}_{n}^{N}=\{\{0, \ldots, n\}\}$ is an $n$-primoid over $N$.

Theorem 3.2. Let $U$ and $U^{\prime}$ be finite sets, let $\mathbf{L}_{n}$ be an n-primoid over $U$ and let $g: U^{\prime} \rightarrow U$ be an onto function. Then a family $\mathbf{L}\left(\mathbf{L}_{n}, g\right)=\left\{\left\{u_{0}, \ldots, u_{n}\right\}\right.$ $\left.\subset U^{\prime}: g\left(\left\{u_{0}, \ldots, u_{n}\right\}\right) \in \mathbf{L}_{n}\right\}$ is nonempty and it is an n-primoid over $U^{\prime}$.

Proof. Take $T^{\prime}=\left\{u_{0}, \ldots, u_{n}\right\} \in \mathbf{L}\left(\mathbf{L}_{n}, g\right)$ and $u \in U^{\prime}$. Notice, that $\left|g\left(T^{\prime}\right)\right|=\left|T^{\prime}\right|$ and the function $g$ is one-to-one on $T^{\prime}$. By the definition of the $n$-primoid, for $g\left(T^{\prime}\right) \in \mathbf{L}_{n}$ and the element $g(u) \in U$ there is exactly one $j \in N$ such that $\left\{g\left(u_{0}\right), \ldots, g\left(u_{j-1}\right), g(u), g\left(u_{j+1}\right), \ldots, g\left(u_{n}\right)\right\} \in \mathbf{L}_{n}$. Hence for $\left\{u_{0}, \ldots, u_{n}\right\} \in \mathbf{L}\left(\mathbf{L}_{n}, g\right)$ and $u \in U^{\prime}$ there is exactly one $j \in N$ such that $\left\{u_{0}, \ldots, u_{j-1}, u, u_{j+1}, \ldots, u_{n}\right\} \in \mathbf{L}\left(\mathbf{L}_{n}, g\right)$.

Corollary 3.3. Let $M_{d}$ be a matroid with a fixed base $\left\{v_{0}, \ldots, v_{n}\right\}$ and let $r$ be the rank function of the matroid $M_{d}$. Denote $\operatorname{span} A=\left\{x \in M_{d}: r(A \cup\{x\})=\right.$ $r(A)\}$. Let $F_{0}=\operatorname{span}\left\{v_{0}\right\}$ and $F_{i}=\operatorname{span}\left\{v_{0}, \ldots, v_{i}\right\} \backslash \operatorname{span}\left\{v_{0}, \ldots, v_{i-1}\right\}$ for $i \in\{1, \ldots, n\}$. Observe, that $M_{d}=\bigcup_{i=0}^{n} F_{i}$ and $F_{i} \cap F_{j}=\emptyset$ for $i \neq j$. Let $g: M_{d} \rightarrow N$ be a function defined by $g(a)=i$ for $a \in F_{i}$. The function $g$ is 
well defined and the family $\mathbf{L}_{n}^{M_{d}}=\left\{\left\{u_{0}, \ldots, u_{n}\right\}: g\left(\left\{u_{0}, \ldots, u_{n}\right\}\right)=N\right\}$ is an n-primoid over $M_{d}$.

Proof. From Theorem 3.2 and Example 3.1 we have $\mathbf{L}_{n}^{M_{d}}=\mathbf{L}\left(\mathbf{L}_{n}^{N}, g\right)$.

Example 3.4 (Bapat, [2, Lemma 4.1]). Let $U=\{-k, \ldots,-1,1, \ldots, k\}$ for some $k \geq n \geq 1$. We define an $n$-primoid $\mathbf{L}_{n}^{k}$ over $U$ as follows: $\left\{u_{0}, \ldots, u_{n}\right\} \in$ $\mathbf{L}_{n}^{k}$ if and only if there exists a permutation $\pi$ such that $\left|u_{\pi(0)}\right| \leq\left|u_{\pi(1)}\right| \leq \ldots \leq$ $\left|u_{\pi(n)}\right|, u_{\pi(i)} \cdot u_{\pi(i+1)}<0$ and if $\left|u_{\pi(i)}\right|=\left|u_{\pi(i+1)}\right|$, then $u_{\pi(i)}<0(i \in N \backslash\{n\})$.

Proof. Take any $\left\{u_{0}, \ldots, u_{n}\right\} \in \mathbf{L}_{n}^{k}$ and any $u \in U$. Without loss of generality we assume that $\pi$ is the identity permutation. If there exists such $k \in N$ that $u=u_{k}$, then $\left\{u_{0}, \ldots, u_{k-1}, u, u_{k+1}, \ldots, u_{n}\right\} \in \mathbf{L}_{n}^{k}$. Otherwise we have three cases:

(a) $|u| \leq\left|u_{0}\right|$. If $u$ and $u_{0}$ have the same signs, then $\left\{u, u_{1}, \ldots, u_{n-1}, u_{n}\right\} \in$ $\mathbf{L}_{n}^{k}$. If they have opposite signs, then $\left\{u, u_{0}, \ldots, u_{n-1}\right\} \in \mathbf{L}_{n}^{k}$.

(b) There exists $i \in\{0, \ldots, n-1\}$ such that $\left|u_{i}\right|<|u| \leq\left|u_{i+1}\right|$. If $u$ and $u_{i}$ have the same signs, then $\left\{u_{0}, \ldots, u_{i-1}, u, u_{i+1}, \ldots, u_{n-1}, u_{n}\right\} \in \mathbf{L}_{n}^{k}$. If they have the opposite signs, then $\left\{u_{0}, \ldots, u_{i}, u, u_{i+2}, \ldots, u_{n}\right\} \in \mathbf{L}_{n}^{k}$.

The case (c) $\left|u_{n}\right|<|u|$ is analogous to the case (a).

From Example 3.4 and Theorem 3.2 we have the following

Theorem 3.5. Let $U$ be a finite set and let for some $k \geq n \geq 1 \mathrm{~g}: U \rightarrow$ $\{-k, \ldots,-1,1, \ldots, k\}$ be an onto function. We define a family $\mathbf{L}_{n}^{g, k}$ as follows: $\left\{u_{0}, \ldots, u_{n}\right\} \in \mathbf{L}_{n}^{g, k}$ if and only if there exists a permutation $\pi$ such that: $\left|g\left(u_{\pi(0)}\right)\right| \leq\left|g\left(u_{\pi(1)}\right)\right| \leq \ldots \leq\left|g\left(u_{\pi(n)}\right)\right|$ and $g\left(u_{\pi(i)}\right) \cdot g\left(u_{\pi(i+1)}\right)<0$ and if $\left|g\left(u_{\pi(i)}\right)\right|=\left|g\left(u_{\pi(i+1)}\right)\right|$, then $g\left(u_{\pi(i)}\right)<0$. Then $\mathbf{L}_{n}^{g, k}$ is an n-primoid over the set $U$.

Proof. Observe that $\mathbf{L}_{n}^{g, k}=\mathbf{L}\left(\mathbf{L}_{n}^{k}, g\right)$.

Example 3.6 (Bapat, [2, Lemma 4.2]). Let $U \subset \mathbb{R}^{n}$ be a finite set with $|U| \geq n+1$ and let $b \in \mathbb{R}^{n}$ be a point, which is not a convex combination of fewer than $n+1$ elements of $U$. If the family $\mathbf{L}_{n}^{b}=\left\{\left\{u_{0}, \ldots, u_{n}\right\}: b \in \operatorname{co}\left\{u_{0}, \ldots, u_{n}\right\}\right\}$ is not empty, then it is an $n$-primoid over $U$.

ExAmple 3.7. Let $A=\left\{a_{0}, \ldots, a_{n}\right\} \subset \mathbb{R}^{n}$ be a set of affinely independent points. Let $m_{A}=\sum_{a \in A} a /|A|$. We say that a function $\pi: 2^{A} \rightarrow \mathbb{R}^{n}$ is in a general position if:

(a) $\pi(B) \in \operatorname{rico}\left\{a_{i}: i \in B\right\}$ for each $B \subset A$,

(b) $m_{A} \notin$ af $\{\pi(\mathbf{D})\}$ for each $\mathbf{D} \subset 2^{A}$ such that $|\mathbf{D}|<|A|$.

A family $\mathbf{D}$ is said to be $\pi$-balanced if $|\mathbf{D}|=|A|, m_{A} \in \operatorname{co}\{\pi(\mathbf{D})\}$ and $\pi$ is in the general position. The set of all $\pi$-balanced families is an $n$-primoid over $P(A)$, 
we denote it by $\mathbf{L}_{n}^{\pi}$. This follows from Theorem 3.2 and Example 3.6, because $\mathbf{L}_{n}^{\pi}=\mathbf{L}\left(\mathbf{L}_{n}^{b}, \pi\right)$ for $b=m_{A}$.

\section{Rank function}

Let $\mathbf{L}_{n}$ be an $n$-primoid over a set $U(|U| \geq n+1)$. A function $\rho: \mathbf{P}(U) \rightarrow$ $\{0, \ldots, n+1\}$ defined by $\rho(A)=\max \left\{|A \cap T|: T \in \mathbf{L}_{n}\right\}$ for $A \in \mathbf{P}(U)$ is called a rank function of $\mathbf{L}_{n}$. Notice that the number $k=n+1-\rho(A)$ is the minimal number such that there exists a set $\left\{u_{1}, \ldots, u_{k}\right\} \subset U$ and the set $A \cup\left\{u_{1}, \ldots, u_{k}\right\}$ contains a complete $n$-simplex.

The rank function of $\mathbf{L}_{n}$ has the following

Properties 4.1. For $A, B \in \mathbf{P}(U)$ :

(a) $\rho(B)=|B|$ for $B \subset A \in \mathbf{L}_{n}$,

(b) $\rho(A) \leq|A|$ for $A \subset U$,

(c) $\rho(A) \leq \rho(B)$ for $A \subset B$,

(d) $\rho(A \cup B) \leq \rho(A)+\rho(B)$.

Proof. Properties (a)-(c) follow directly from the definition of the $n$-primoid. For the property (d) consider $T \in \mathbf{L}_{n}$ such that $|T \cap(A \cup B)|=\rho(A \cup B)$. Then $|T \cap(A \cup B)| \leq|T \cap A|+|T \cap B|$ and $|T \cap A| \leq \rho(A),|T \cap B| \leq \rho(B)$. Hence $\rho(A \cup B) \leq \rho(A)+\rho(B)$.

Observe that a function defined by:

(a) $\rho(A)=|A|$ for $A \subset U$ is the rank function of $\mathbf{L}_{n}^{N}$ (see Example 3.1),

(b) $\rho(A)=|\{g(u): u \in A\}|$ for $A \subset U$ is the rank function of the $n$-primoid $\mathbf{L}\left(\mathbf{L}_{n}^{N}, g\right)$ over $U$ (see Theorem 3.2),

(c) $\rho_{\mathbf{L}\left(\mathbf{L}_{n}, g\right)}(A)=\rho_{\mathbf{L}_{n}}(\{g(u): u \in A\})(A \subset U)$ is the rank function of the $n$-primoid $\mathbf{L}\left(\mathbf{L}_{n}, g\right)$ for the rank function $\rho_{\mathbf{L}_{n}}$ of the $n$-primoid $\mathbf{L}_{n}$ (see Theorem 3.2).

Let $\rho$ be the rank function of $\mathbf{L}_{n}$. A set $R \subset U$ is a maximal set of the rank $k(k \in \mathbb{N})$ if $\rho(R)=k$ and for each $u \in U \backslash R, \rho(R \cup\{u\})=k+1$. Now we define a subset sp $A \subset U$ spanned by elements of $A \subset U$ in the sense of the $n$-primoid $\mathbf{L}_{n}$. For $A \subset U$ a spanned set by a set $A$ is $\operatorname{sp} A=\bigcap\{R: A \subset R \subset$ $U$ and $R$ is a maximal set of the $\operatorname{rank} \rho(A)\}$.

Observe that the spanned set by a set $A$ is defined:

(a) $\operatorname{sp} A=A$ for the $n$-primoid $\mathbf{L}_{n}^{N}$ (see Example 3.1),

(b) $\operatorname{sp} A=\{u: g(u) \in g(A)\}$ for the $n$-primoid $\mathbf{L}\left(\mathbf{L}_{n}^{N}, g\right)$,

(c) $\operatorname{sp}_{\mathbf{L}\left(\mathbf{L}_{n}, g\right)} A=\left\{u: g(u) \in \operatorname{sp}_{L_{n}} g(A)\right\}$ for the $n$-primoid $\mathbf{L}\left(\mathbf{L}_{n}, g\right)$, where $\operatorname{sp}_{\mathbf{L}_{n}}$ is in the sense of the $n$-primoid $\mathbf{L}_{n}$.

Properties 4.2. For the n-primoid $\mathbf{L}_{n}$ and $A \subset U$ :

(a) $A \subset \operatorname{sp} A$, 
(b) $\rho(A)=\rho(\operatorname{sp} A)$,

(c) if a set $A$ contains a complete set, then $\operatorname{sp} A=U$.

Proof. The property (a) is obvious.

(b) Observe that $\rho(A) \leq \rho(\operatorname{sp} A)$ because of (a) and $\rho(A) \leq \rho(B)$ for $A \subset B$. On the other hand $\rho(A) \geq \rho(\operatorname{sp} A)$, because $\operatorname{sp} A$ is the intersection of the sets of the $\operatorname{rank} \rho(A)$.

(c) The only maximal set with the $\operatorname{rank} \rho(A)=n+1$ is $U$.

Theorem 4.3. Let $T=\left\{u_{0}, \ldots, u_{n}\right\} \in \mathbf{L}_{n}, u \in \operatorname{sp}\left\{u_{i}: i \in M\right\},(M \subset N)$ and $T^{\prime}=\left\{u_{0}, \ldots, u_{j-1}, u, u_{j+1}, \ldots, u_{n}\right\} \in \mathbf{L}_{n}$, then $j \in M$.

Proof. From Properties 4.1(a) and 4.2(b) $\rho\left(\operatorname{sp}\left\{u_{i}: i \in M\right\}\right)=|M|$. If $j \notin M$, then $\operatorname{sp}\left\{u_{i}: i \in M\right\} \cap T^{\prime}=\left\{u_{i}: i \in M\right\} \cup\{u\}$ and we get $\rho\left(\operatorname{sp}\left\{u_{i}: i \in\right.\right.$ $M\}) \geq|M|+1$.

Corollary 4.4. If $\left\{u_{0}, \ldots, u_{n}\right\} \in \mathbf{L}_{n}$ and $u \in \operatorname{sp}\left\{u_{i}\right\}$ for some $i \in N$, then $\left\{u_{0}, \ldots, u_{i-1}, u, u_{i+1}, \ldots, u_{n}\right\} \in \mathbf{L}_{n}$.

Corollary 4.5. If $\left\{u_{0}, \ldots, u_{n}\right\} \in \mathbf{L}_{n}$ and $u_{i}^{\prime} \in \operatorname{sp}\left\{u_{i}\right\}$ for all $i \in N$, then $\left\{u_{0}^{\prime}, \ldots, u_{n}^{\prime}\right\} \in \mathbf{L}_{n}$.

\section{Main theorem}

Let $n \in \mathbb{N}$ and $\mathbf{C}$ be a nonempty finite family of $n$-simplexes of a set $V$. Let $\mathbf{L}_{n}$ be an $n$-primoid over the set $U$ and let $x \in U$ be a fixed element. Let $l: V \rightarrow U$ be a fixed labelling function.

A simplex $S \in \mathbf{C}$ is called completely labelled simplex (for short c.l.) if $l(S) \in \mathbf{L}_{n}$. For $v \in S$ a pair $(S, v)$ is called $x$-labelled pair (for short $x$-l.) if $l(S \backslash\{v\}) \cup\{x\} \in \mathbf{L}_{n}$.

A completely labelled simplex $S \in \mathbf{C}$ is adjacent by a primal pivot to $x$ labelled pair $\left(S^{\prime}, v^{\prime}\right)$ if and only if $S=S^{\prime}\left(S^{\prime} \in \mathbf{C}, v^{\prime} \in S^{\prime}\right)$.

Two different $x$-labelled pairs $(S, v)$ and $\left(S^{\prime}, v^{\prime}\right)\left(S, S^{\prime} \in \mathbf{C}, v \in S, v^{\prime} \in S^{\prime}\right)$ are adjacent by a primal pivot if and only if $S=S^{\prime}$.

LEMMA 5.1.

(a) Every completely labelled simplex is adjacent by a primal pivot to a single $x$-labelled pair.

(b) Every x-labelled pair is adjacent by a primal pivot either to a single $x$-labelled pair or to a single completely labelled simplex.

Proof. (a) Let $S$ be a completely labelled simplex. For $l(S) \in \mathbf{L}_{n}$ and $x \in U$ there is exactly one $u \in l(S)$ such that $l(S) \backslash\{u\} \cup\{x\} \in \mathbf{L}_{n}$. Since $|l(S)|=|S|$ there exists exactly one $v \in S$ such that $l(v)=u$. Therefore 
$l(S) \backslash\{u\}=l(S \backslash\{v\})$. Hence $(S, v)$ is the $x$-labelled pair and the only one that is adjacent by a primal pivot to the completely labelled simplex $S$.

(b) Take the $x$-labelled pair $(S, v)$. For $l(S \backslash\{v\}) \cup\{x\} \in \mathbf{L}_{n}$ and $l(v) \in U$ there exists exactly one element $u \in l(S \backslash\{v\}) \cup\{x\}$ such that $(l(S \backslash\{v\}) \cup$ $\{x\}) \backslash\{u\} \cup\{l(v)\} \in \mathbf{L}_{n}$. If $u=x$, then the simplex $S$ is completely labelled and it is the only completely labelled simplex adjacent to the pair $(S, v)$ by a primal pivot. If $u \neq x$, then $u \in l(S \backslash\{v\})$ so there exists $v^{\prime} \in S \backslash\{v\}$ such that $u=l\left(v^{\prime}\right) . \quad(l(S \backslash\{v\}) \cup\{x\}) \backslash\left\{l\left(v^{\prime}\right)\right\} \cup\{l(v)\}=l\left(S \backslash\left\{v^{\prime}\right\}\right) \cup\{x\}$ and the pair $\left(S, v^{\prime}\right)$ is $x$-l. and the only $x$-l. pair adjacent to $(S, v)$.

THEOREM 5.2. Let $\mathbf{C}$ be a nonempty finite family of n-simplexes over $V$. Let $\mathbf{L}_{n}$ be an n-primoid over the set $U$. Let $l: V \rightarrow U$ be a fixed labelling function and let $x \in U$ be a fixed element. Then the number of c.l. simplexes is equal to the number of $x$-l. pairs modulo 2.

PRoOF. It follows directly from Lemma 5.1.

\section{Case of the pseudomanifold}

An $n$-dimensional pseudomanifold (or n-pseudomanifold) is a finite family C of $n$-simplexes over the set $V$ having the following property: every $(n-1)$ simplex over $V$ is a subset of at most two $n$-simplexes of C. An $(n-1)$-simplex $S$ over the set $V$, which is a subset of exactly one element of an $n$-pseudomanifold $\mathbf{C}$ is called a boundary $(n-1)$-simplex of $\mathbf{C}$. Let $\mathbf{L}_{n}$ be an $n$-primoid over the set $U$. For $x \in U, S \in \mathbf{C}$ and $v \in S$ an $x$-labelled pair $(S, v)$ is a boundary $x$-labelled pair if $S \backslash\{v\}$ is a boundary simplex of $\mathbf{C}$.

Theorem 6.1. Let $\mathbf{C}$ be an n-pseudomanifold over $V, \mathbf{L}_{n}$ be an n-primoid over the set $U$ and $x \in U$ be a fixed element. Let $l: V \rightarrow U$ be a fixed labelling function. Then the number of c.l. simplexes is equal to the number of boundary $x$-l. pairs modulo 2 .

Proof. Take an $x$-l. pair $(S, v)(S \in \mathbf{C}, v \in S)$ which is not a boundary $x$-labelled pair. $S \backslash\{v\}$ is not a boundary simplex of $\mathbf{C}$. Because $\mathbf{C}$ is the pseudomanifold, then there is exactly one $S^{\prime} \in \mathbf{C}$ and exactly one $v^{\prime} \in S^{\prime}$ such that $S \backslash\{v\}=S^{\prime} \backslash\left\{v^{\prime}\right\}$. Notice that the pair $\left(S^{\prime}, v^{\prime}\right)$ is also the $x$-l. pair which is not a boundary $x$-labelled pair. Hence $x$-l. pairs which are not boundary $x$-labelled pairs have no influence on the number of $x$-1. pairs modulo 2. Thus Theorem 6.1 follows from Theorem 5.2.

From Theorem 6.1 we have:

Corollary 6.2.

(a) For $V=U$ and $l$ is the identity function we get a special case of Todd's Theorem 5.3 ([21]). 
(b) For $\mathbf{L}_{n}=\mathbf{L}\left(\mathbf{L}_{n}^{N}, g\right)$ we get Gould and Tolle's Theorem 5.2 .5 ([9]).

(c) For $\mathbf{L}_{n}=\mathbf{L}_{n}^{M_{d}}$ we get Lovász's Theorem ([16], see also Kryński [14]).

(d) For $\mathbf{L}_{n}=\mathbf{L}_{n}^{k}$ we get Fan's Combinatorial Lemma ([6]).

\section{Case of the geometric simplex}

Let $\left\{d_{0}, \ldots, d_{n}\right\}$ be a fixed set of affinely independent vectors in $\mathbb{R}^{n}$. For $M \subset N$ we denote $\Delta^{M}=\operatorname{co}\left\{d_{i}: i \in M\right\}$. Let Tr be a triangulation of $\Delta^{N}$. For every simplex $\delta$ in $\operatorname{Tr}$ let $V(\delta)$ denote the set of its vertices and $V=V(\operatorname{Tr})$. The family $\mathbf{C}=\{V(\delta): \delta \in \operatorname{Tr}\}$ is a pseudomanifold. Let $\mathbf{L}_{n}$ be an $n$-primoid over the set $U$.

Let $l: V \rightarrow U$ be a labelling function. We say that $l$ satisfies a $\rho$-boundary condition if:

(1) $\left\{l\left(d_{0}\right), \ldots, l\left(d_{n}\right)\right\} \in \mathbf{L}_{n}$,

(2) $\rho\left(l\left(V \cap \Delta^{M}\right)\right)=|M|$ for any $M \subset N$.

Theorem 7.1. Let $\operatorname{Tr}$ be a triangulation of $\Delta^{N}, \mathbf{C}=\{V(\delta): \delta \in \operatorname{Tr}\}$, $V=V(\operatorname{Tr})$ and $\mathbf{L}_{n}$ be an n-primoid over a set $U$. Let $l: V \rightarrow U$ be a labelling function satisfying the $\rho$-boundary condition. Then the number of c.l. simplexes is odd. In particular there is at least one c.l. simplex.

Proof. We may assume that the triangulation Tr does not subdivide the boundary simplexes $\Delta^{N \backslash\{i\}}$ for any $i \in N$. If the assumption is not satisfied we may embed $\Delta^{N}$ in a larger simplex using the Scarf method ([17, p. 192]): for a larger simplex $\widetilde{\Delta}^{N}=\operatorname{co}\left\{\widetilde{d}_{0}, \ldots, \widetilde{d}_{n}\right\}$ containing $\Delta^{N}$ we join every vertex $v \in V \cap \Delta^{N \backslash\{i\}}$ with the vertex $\widetilde{d}_{i+1}\left(\widetilde{d}_{n+1}=\widetilde{d}_{0}\right)$. So defined triangulation of $\widetilde{\Delta}^{N}$ we denote by $\widetilde{\operatorname{Tr}}$. We define a labelling $\widetilde{l}$ as an extension of $l$ on $V(\widetilde{T r})$ by $\widetilde{l}\left(\widetilde{d}_{i}\right)=l\left(d_{i}\right)$ for all $i \in N$.

We proof that in this new triangulation $\widetilde{\operatorname{Tr}}$ of $\widetilde{\Delta}^{N}$ and the labelling $\widetilde{l}$ there is no new c.l. simplexes. For a simplex $\delta \in \widetilde{T r} \backslash \operatorname{Tr}$, let $V(\delta)=S=\left\{w_{i} \in \Delta^{M_{S}} \cap S\right.$ : $\left.i \in M_{S}\right\} \cup\left\{\widetilde{d}_{i+1}: i \notin M_{S}\right\}\left(M_{S} \subset N\right)$. There exists $k \in N$ such that $k \notin M_{S}$ and $k+1 \in M_{S}(k+1$ is taken $\bmod (n+1))$. Because the labelling $l$ satisfies the $\rho$-boundary condition we have $\rho\left(\left\{\widetilde{l}\left(w_{i}\right): i \in M_{S}\right\} \cup \widetilde{l}\left(\widetilde{d}_{k+1}\right)\right) \leq\left|M_{S}\right|$. From Property 4.1(d) we have

$$
\begin{aligned}
\rho(\widetilde{l}(S)) & =\rho\left(\left\{l\left(w_{i}\right): i \in M_{S}\right\} \cup\left\{l\left(\widetilde{d}_{k+1}\right)\right\} \cup\left\{l\left(\widetilde{d}_{j+1}: j \notin M_{S}, j \neq k\right)\right\}\right) \\
& \leq\left|M_{S}\right|+\left(n+1-\left|M_{S}\right|-1\right)=n
\end{aligned}
$$

and thus $S$ is not a c.l. simplex. Obviously $\widetilde{l}$ also satisfies the $\rho$-boundary condition.

By Theorem 6.1, for a fixed $x \in U$ the number of c.l. simplexes is equal to the number of the boundary $x$-l. pairs modulo 2 . Now observe that there is exactly one boundary $x$-l. pair. For $l\left(\left\{d_{0}, \ldots, d_{n}\right\}\right) \in \mathbf{L}_{n}$ there is exactly one $l\left(d_{i}\right)$ 
such that $l\left(\left\{d_{0}, \ldots, d_{i-1}, d_{i+1}, \ldots, d_{n}\right\}\right) \cup\{x\} \in \mathbf{L}_{n}(i \in N)$. Hence there exists exactly one simplex $\delta \in \operatorname{Tr}$ with $V(\delta)=S=\left\{d_{0}, \ldots, d_{i-1}, d_{i+1}, \ldots, d_{n}, v\right\} \in \mathbf{C}$, where $v \in \operatorname{ri} \Delta^{N}$ and $(S, v)$ is boundary $x$-labelled pair.

We say that $l$ satisfies an sp -boundary condition for $\left\{u_{0}, \ldots, u_{n}\right\} \in \mathbf{L}_{n}$ if:

(sp) for every $M \subset N$ and for every $v \in\left(V \cap \Delta^{M}\right), l(v) \in \operatorname{sp}\left\{u_{i}: i \in M\right\}$.

Theorem 7.2. Let $\operatorname{Tr}$ be a triangulation of $\Delta^{N}, \mathbf{C}=\{V(\delta): \delta \in \operatorname{Tr}\}$, $V=V(\operatorname{Tr})$ and $\mathbf{L}_{n}$ be an n-primoid over a set $U$. Let $l: V \rightarrow U$ be a labelling function, such that $\left\{l\left(d_{0}\right), \ldots, l\left(d_{n}\right)\right\} \in \mathbf{L}_{n}$ and let $l$ satisfy the sp-boundary condition for $\left\{l\left(d_{0}\right), \ldots, l\left(d_{n}\right)\right\}$. Then the number of c.l. simplexes is odd. In particular there is at least one c.l. simplex.

Proof. By the sp-boundary condition $l\left(V \cap \Delta^{M}\right) \subset \operatorname{sp}\left\{l\left(d_{j}\right): j \in M\right\}$ for any $M \subset N$. From Properties 4.1(c) we have $\rho\left(l\left(V \cap \Delta^{M}\right)\right) \leq \rho\left(\operatorname{sp}\left\{l\left(d_{j}\right): j \in\right.\right.$ $M\})$ for any $M \subset N$. On the other hand $l\left(d_{j}\right) \in l\left(V \cap \Delta^{M}\right)$ for $j \in M$ so $\left\{l\left(d_{j}\right): j \in M\right\} \subset l\left(V \cap \Delta^{M}\right)$ and $\rho\left(\left\{l\left(d_{j}\right): j \in M\right\}\right) \leq \rho\left(\left\{l\left(d_{j}\right) \in l\left(V \cap \Delta^{M}\right)\right\}\right)$. By Property $4.2(\mathrm{~b}) \rho\left(\operatorname{sp}\left\{l\left(d_{j}\right): j \in M\right\}\right)=\rho\left(\left\{l\left(d_{j}\right): j \in M\right\}\right)=|M|$. Hence $\rho\left(l\left(V \cap \Delta^{M}\right)=\rho\left(\left\{l\left(d_{j}\right): j \in M\right\}\right)=|M|\right.$ for $M \subset N$ and the conditions of Theorem 7.1 are satisfied.

From Theorem 7.2 we have:

\section{Corollary 7.3.}

(a) For $\mathbf{L}_{n}=\mathbf{L}_{n}^{N}$ we get Sperner's Lemma ([19]).

(b) For $\mathbf{L}_{n}=\mathbf{L}_{n}^{M_{d}}$ we get Lovász's Corollary ([16], see also Kryński [14]).

(c) For $\mathbf{L}_{n}=\mathbf{L}_{n}^{\pi}$ we get Shapley's Lemma 7.2 ([18]).

Let $\operatorname{Tr}$ be a triangulation of $\Delta^{N}$. Let $l: V(\operatorname{Tr}) \rightarrow \mathbb{R}^{n}$ be a labelling and let $b \in \operatorname{ri} \Delta^{N}$ be a point which is not a convex combination of fewer than $n+1$ elements of $l(V)$. A simplex $\delta \in \operatorname{Tr}$ is called b-balanced if $b \in \operatorname{col} l(V(\delta))$.

TheOREM 7.4. Let $\operatorname{Tr}$ be a triangulation of $\Delta^{N}=\operatorname{co}\left\{d_{0}, \ldots, d_{n}\right\}$ and $\mathbf{C}=$ $\{V(\delta): \delta \in \operatorname{Tr}\}, V=V(\operatorname{Tr})$. Let $l: V \rightarrow \mathbb{R}^{n}$ be a labelling such that: for any $M \subset N$, if $v \in V \cap \Delta^{M}$, then $l(v) \in$ af $\left\{d_{i}: i \in M\right\}$. Let $b \in \operatorname{ri} \Delta^{N}$ be a point which is not a convex combination of fewer than $n+1$ elements of $l(V)$. Then the number of b-balanced simplexes is odd. In particular there is at least one b-balanced simplex.

Proof. The family $\mathbf{L}_{n}^{b}=\{A \subset l(V):|A|=n+1, b \in \operatorname{co} A\}$ is an $n$-primoid (see Example 3.7) and $\left\{d_{0}, \ldots, d_{n}\right\} \in \mathbf{L}_{n}^{b}$. Any set $T \in \mathbf{L}_{n}^{b}$ is affinely independent thus $\left|T \cap l\left(V \cap \Delta^{M}\right)\right| \leq|M|$ for any $M \subset N$ and $\rho\left(l\left(V \cap \Delta^{M}\right)\right)|\leq| M \mid$. Therefore our theorem follows from Theorem 7.1.

For a fixed element $\left\{u_{0}, \ldots, u_{n}\right\}$ of an $n$-primoid $\mathbf{L}_{n}$ over a set $U$ a family of closed sets $\left\{C_{u}: u \in U\right\}$ is the KKM covering ([13]) if 


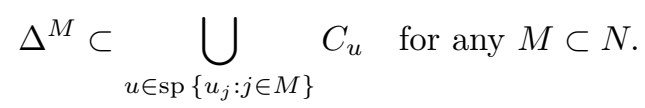

ThEOREM 7.5. Let $\mathbf{L}_{n}$ be an n-primoid over a set $U$ and $\mathbf{F}=\left\{C_{u}: u \in U\right\}$ be a family of closed sets such that $\mathbf{F}$ is the KKM covering for some $\left\{u_{0}, \ldots, u_{n}\right\}$ $\in \mathbf{L}_{n}$. Then there exist an element $\left\{w_{0}, \ldots, w_{n}\right\} \in \mathbf{L}_{n}$ and a point $z \in \Delta^{N}$ such that $z \in \bigcap_{i=0}^{n} C_{w_{i}}$.

Proof. Let $\operatorname{Tr}^{k}(k \in \mathbb{N})$ be a sequence of a triangulations of $\Delta^{N}$ with the mesh tending to zero, when $k$ tends to infinity. We define a labelling $l_{k}$ of the vertices of $\operatorname{Tr}^{k} k \in \mathbb{N}$ in the following way: $l\left(d_{i}\right)=u_{i}$ for $i \in N$ and for $v \in V\left(\operatorname{Tr}^{k}\right)=V_{k}\left(v \notin\left\{d_{0}, \ldots, d_{n}\right\}\right)$ let $l_{k}(v)=u$ for some $u$ such, that $v \in C_{u}$ and if $v \in \Delta^{M}$, then $u \in \operatorname{sp}\left\{l_{k}\left(\Delta^{M} \cap V_{k}\right)\right\}$. Since $\left\{C_{u}: u \in U\right\}$ is KKM covering for $\left\{u_{0}, \ldots, u_{n}\right\}$, then the labelling $l_{k}$ is well defined and it satisfies the sp-boundary condition for $\left\{l\left(d_{0}\right), \ldots, l\left(d_{n}\right)\right\}=\left\{u_{0}, \ldots, u_{n}\right\}$. It follows from Theorem 7.2 that there exist a c.l. simplex $S_{k}=\left\{v_{0}^{k}, \ldots, v_{n}^{k}\right\}$. Hence for $i \in N$ $v_{i}^{k} \in C_{l_{k}\left(v_{i}^{k}\right)}$. Because the mesh of $\operatorname{Tr}_{k}$ tends to zero, there exist $z \in \Delta^{N}$ and a subsequence of $v_{i}^{k}$ which converges to $z$ for each $i \in N$. Since $C_{u}$ is a closed set for $u \in U$, then $z \in C_{l_{k_{0}}\left(v_{i}^{k}\right)}$ for $i \in N$ and the proof is complete.

From Theorem 7.5 we have:

\section{Corollary 7.6.}

(a) For $\mathbf{L}_{n}=\mathbf{L}_{n}^{N}$ we get the classic KKM Theorem ([13]).

(b) For $\mathbf{L}_{n}=\mathbf{L}_{n}^{M_{d}}$ we get Idzik's Theorem 5 ([12]).

(c) For $\mathbf{L}_{n}=\mathbf{L}_{n}^{\pi}$ we get Shapley's Lemma 7.3 ([18]).

TheOrem 7.7. Let $\mathbf{F}=\left\{C_{u}: u \in U \subset \mathbb{R}^{n}\right\}$ be a family of closed sets such that for any $M \subset N, \Delta^{M} \subset \bigcup_{u \in \operatorname{af}\left\{d_{i}: i \in M\right\}} C_{u}$. Let $b \in \operatorname{ri} \Delta^{N}$ be a point which is not a convex combination of fewer than $n+1$ elements of $U$. Then there exist a set $\left\{w_{0}, \ldots, w_{n}\right\} \subset U$ such that $b \in \operatorname{co}\left\{w_{0}, \ldots, w_{n}\right\}$ and a point $z \in \Delta^{N}$ such that $z \in \bigcap_{i=0}^{n} C_{w_{i}}$

Proof. It is analogous to the proof of Theorem 7.5. Theorem 7.4 is used instead of Theorem 7.2. if:

We say that $l$ satisfies a dual sp-boundary condition for $\left\{u_{0}, \ldots, u_{n}\right\} \in \mathbf{L}_{n}$

(dsp) for every $M \subset N, M \neq N$ and for every $v \in V \cap \operatorname{ri} \Delta^{M}, l(v) \in \operatorname{sp}\left\{u_{i}:\right.$ $i \notin M\}$.

TheOREM 7.8. Let $\operatorname{Tr}$ be a triangulation of $\Delta^{N}, \mathbf{C}=\{V(\delta): \delta \in \operatorname{Tr}\}$, $V=V(\operatorname{Tr})$ and for any $M \subset N$, ri $\Delta^{M} \cap V \neq \emptyset$. Let $\mathbf{L}_{n}$ be an n-primoid over a set $U$. For a labelling $l: V \rightarrow U$ satisfying the dual $\mathrm{sp}$-boundary condition for 
some $\left\{u_{0}, \ldots, u_{n}\right\} \in \mathbf{L}_{n}$ the number of c.l. simplexes is odd. In particular there is at least one c.l. simplex.

Proof. We imbed $\Delta^{N}$ in a larger simplex $\widetilde{\Delta}^{N}=\operatorname{co}\left\{\widetilde{d}_{0}, \ldots, \widetilde{d}_{n}\right\}$ containing $\Delta^{N}$ joining every vertex $v \in V \cap \Delta^{N \backslash\{i\}}$ with the vertex $\widetilde{d}_{i}$, (similarly to Scarf method in [17, p. 192]). So defined triangulation of $\widetilde{\Delta}^{N}$ we denote by $\widetilde{T r}$. We define a labelling $\widetilde{l}$ as an extension of $l$ on $V(\widetilde{\operatorname{Tr}})$ by $\widetilde{l}\left(\widetilde{d}_{i}\right)=u_{i}$ for all $i \in N$. We proof that there is no c.l. simplex in $\widetilde{T r} \backslash \operatorname{Tr}$. Consider a simplex $\delta \in \widetilde{T r} \backslash \operatorname{Tr}$. Let $V(\delta)=S=\left\{w_{i} \in \Delta^{M_{S}} \cap S: i \in M_{S}\right\} \cup\left\{\widetilde{d}_{i}: i \notin M_{S}\right\},\left(M_{S} \subset N\right)$. There exists $j \in M_{S}$ such that $w_{j} \in \Delta^{M_{S}}$ and $w_{j} \notin \Delta^{M}$ for any proper subset $M$ of the set $M_{S}$, because $\operatorname{Tr}$ divides $\Delta^{M_{S}}$.

From the dual sp-boundary condition $l\left(w_{j}\right) \in \operatorname{sp}\left\{u_{i}: i \notin M_{S}\right\}$. Hence from Properties 4.2(b) $\rho\left(l\left(\left\{w_{j}\right\} \cup\left\{u_{i}: i \notin M_{S}\right\}\right)=\left|M_{S}\right|\right.$ and from Properties 4.1 $l(S) \leq n$. This means $S$ is not completely labelled. Notice, that $\widetilde{l}$ fulfills the $\rho$-boundary condition and by Theorem 7.1 we get our theorem.

For a fixed element $\left\{u_{0}, \ldots, u_{n}\right\}$ of an $n$-primoid $\mathbf{L}_{n}$ over a set $U$ a family of closed sets $\left\{C_{u}: u \in U\right\}$ is the dual KKM covering ([10]) if:

(1) $\Delta^{N} \subset \bigcup_{u \in U} C_{u}$.

(2) $\Delta^{M} \subset \bigcup_{u \in \operatorname{sp}\left\{u_{j}: j \notin M\right\}} C_{u}$ for any proper subset $M$ of the set $N$.

TheOREM 7.9. Let $\mathbf{L}_{n}$ be an n-primoid over a set $U$ and $\mathbf{F}=\left\{C_{u}: u \in U\right\}$ be a family of closed sets such that $\mathbf{F}$ is the dual KKM covering for $\left\{u_{0}, \ldots, u_{n}\right\} \in$ $\mathbf{L}_{n}$. Then there exists an element $\left\{w_{0}, \ldots, w_{n}\right\} \in \mathbf{L}_{n}$ and a point $z \in \Delta^{N}$ such that $z \in \bigcap_{i=0}^{n} C_{w_{i}}$.

Proof. It is analogous to the proof of Theorem 7.5. Theorem 7.8 is used instead of Theorem 7.2.

Theorem 7.10. Let $\operatorname{Tr}$ be a triangulation of $\Delta^{N}=\operatorname{co}\left\{d_{0}, \ldots, d_{n}\right\}, \mathbf{C}=$ $\{V(\delta): \delta \in \operatorname{Tr}\}$ and for any $M \subset N$, ri $\Delta^{M} \cap V(\delta) \neq \emptyset$ for some $\delta \in \operatorname{Tr}$. Let $l: V(\operatorname{Tr}) \rightarrow \mathbb{R}^{n}$ be a labelling such that: for any $M \subset N, M \neq N$, and for any $v \in V(\operatorname{Tr}) \cap \operatorname{ri} \Delta^{M}, l(v) \in \operatorname{af}\left\{d_{i}: i \notin M\right\}$. Let $b \in \operatorname{ri} \Delta^{N}$ be a point which is not a convex combination of fewer than $n+1$ elements of $l(V)$. Then the number of b-balanced simplexes is odd. In particular there is at least one b-balanced simplex.

Proof. It is analogous to proof of Theorem 7.8 and follows from Theorem 7.1.

Theorem 7.11. Let $\mathbf{F}=\left\{C_{u}: u \in U \subset \mathbb{R}^{n}\right\}$ be a family of closed sets such that $\Delta^{N} \subset \bigcup_{u \in U} C_{u}$ and for any $M \subset N, N \neq M, \Delta^{M} \subset \bigcup_{u \in \operatorname{af}\left\{d_{i}: i \notin M\right\}} C_{u}$. Let $b \in \mathrm{ri} \Delta^{N}$ be a point which is not a convex combination of fewer than $n+1$ elements of $U$. Then there exist a set $\left\{w_{0}, \ldots, w_{n}\right\} \subset U$ such that $b \in \operatorname{co}\left\{w_{0}, \ldots, w_{n}\right\}$ and a point $z \in \Delta^{N}$ such that $z \in \bigcap_{i=0}^{n} C_{w_{i}}$ 
Proof. It is analogous to the proof of Theorem 7.5. Theorem 7.10 is used instead of Theorem 7.2.

REMARK 7.12. In Theorems 7.4, 7.10 and Theorems 7.2, 7.8 applied to the $n$-primoid $\mathbf{L}_{n}^{b}$ the condition: $b \in \operatorname{ri} \Delta^{N}$ is a point which is not a convex combination of fewer than $n+1$ elements of $l(V)$ is essential. However omitting this condition we may still prove that there exists at least one $b$-balanced simplex (not necessarily the odd number of such simplexes). To prove this we take a sequence of points $b_{k}$, which converges to the point $b$ and $b_{k}$ is not a convex combination of fewer that $n+1$ elements of $l(V)$ for any $k \in \mathbb{N}$. Therefore in our Theorems 7.7, 7.11 and Theorems 7.5, 7.9 applied to the $n$-primoid $\mathbf{L}_{n}^{b}$ the condition: $b \in \operatorname{ri} \Delta^{N}$ is a point which is not a convex combination of fewer than $n+1$ elements of $l(V)$ can be omitted. Hence we get the Ichiishi and Idzik Theorem 1.2 in [11] and a special case of the van der Laan, Talman and Yang Theorem 3.1 in [15] (formulated for the simplex).

\section{REFERENCES}

[1] P. Alexandroff and B. Pasynkoff, Elementary proof of the essentiality of the identity mapping of a simplex, Uspiehi Mat. Nauk 12 (1957), 175-179. (Russian)

[2] R. B. BApAt, A generalization of a theorem of Ky Fan on simplicial maps, J. Combin. Theory Ser. A 29 (1978), 32-38.

[3] - A constructive proof of permutation-based generalization of Sperner's lemma, Math. Programming 44 (1988), 113-120.

[4] L. E. J. Brouwer, Über Abbildung von Mannigfaltigkeiten, Math. Ann. 71 (1912), $97-115$.

[5] I. A. Cohen, On the Sperner Lemma, J. Combin. Theory 2 (1967), 585-587.

[6] K. FAN, A generalization of Tucker's combinatorial lemma with topological applications, Ann. of Math. 52 (1952), 431-437.

[7] Simplicial maps from orientable n-pseudomanifold into $S^{n}$ with octahedral triangulation, J. Combin. Theory 2 (1967), 588-602.

[8] _ A combinatorial property of pseudomanifolds and covering properties of simplexes, J. Math. Anal. Appl. 31 (1970), 68-80.

[9] F. J. Gould and J. W. Tolle, A unified approach to complementarity in optimalization, Discrete Math. 7 (1974), 225-271.

[10] T. Ichishi, Alternative version of Shapley's theorem on closed coverings of a simplex, Proc. Amer. Math. Soc. 104 (1988), 759-763.

[11] T. ICHIISHI AND A. IDZIK, Theorems on closed coverings of simplex and their applications to cooperative game theory, J. Math. Anal. Appl. 146 (1990), 259-270.

[12] A. IDZIK, KKM Theorem and Matroids, ICS PAS Reports, vol. 693, 1990.

[13] B. Knaster, C. Kuratowski and S. Mazurkiewicz, Ein Beweis des Fixpunktsatzes für n-dimentiosionale Simplexe, Fund. Math. 14 (1964), 132-137.

[14] W. KRyŃski, Remarks on matroids and Sperner's lemma, European J. Combin. 11 (1990), 485-488.

[15] G. VAN DER LAAN, D. TALMAN AND Z. YANG, Existence of balanced simplices on polytopes, J. Combin. Theory Ser. A 96 (2001), 288-302. 
[16] L. LovÁsz, Matroids and Sperner's lemma, European J. Combin. 2 (1981), 65-66.

[17] H. Scarf (with the collaboration of T. Hansen), Computation of Economics Equilibria (1973), Yale University Press, New Haven.

[18] L. S. Shapley, On balanced games without side payments, Mathematical Programming (T. C. Hu and S. M. Robinson, eds.), Academic Press, New York, 1973, pp. 261-290.

[19] E. Sperner, Neuer Beweis für die Invarianz der Dimensionszahl und des Gebiets, Abh. Math. Sem. Univ. Hamburg 6 (1928), 265-272.

[20] S. PARK, The Knaster-Kuratowski-Mazurkiewicz theorem and almost fixed points, Topol. Methods Nonlinear Anal. 16 (2000), 195-200.

[21] M. J. Todd, A generalized complementary pivoting algorithm, Math. Programming 6 (1974), 243-263

Manuscript received August 7, 2003

ADAM IDZIK

Akademia Świętokrzyska

Świętokrzyska 15

25-406 Kielce, POLAND

and

Institute of Computer Science

Polish Academy of Sciences

Ordona 21

01-237 Warsaw, POLAND

E-mail address: adidzik@ipipan.waw.pl

Konstanty JunOSZA-SzaniaWski

Warsaw University of Technology

Pl. Politechniki 1

00-661 Warsaw, POLAND

E-mail address: junoszak@prioris.mini.pw.edu.pl

TMNA : Volume $22-2003-\mathrm{N}^{\circ} 2$ 